\title{
Restorative proctocolectomy with ileal reservoir: pathological and histochemical study of mucosal biopsy specimens
}

\author{
N A SHEPHERD, ${ }^{*} \S$ J R JASS, ${ }^{*} \ddagger \S$ I DUVAL, $\ddagger$ R L MOSKOWITZ, $\dagger$ R J NICHOLLS, $\ddagger$ \\ B C MORSON* \\ From the Departments of * Pathology, $\dagger$ Surgery, and the $\ddagger$ Imperial Cancer Research Fund Colorectal Cancer \\ Unit, St Mark's Hospital, London, and the §Department of Histopathology, St Bartholomew's Hospital, London
}

SUMmaRY Mucosal biopsy specimens from the ileal reservoirs of 92 patients who had undergone restorative proctocolectomy (12 with familial adenomatous polyposis, 78 with ulcerative colitis, and two with functional bowel disease) were studied. Chronic inflammation was found in almost all, as was villous atrophy of varying severity. Other changes included pyloric metaplasia and mucosal prolapse. Acute inflammatory changes and ulceration were less common but, when present, corresponded to the clinical condition of "pouchitis". A grading system was devised to score acute and chronic inflammatory changes. There was a significant increase in acute inflammatory scores in ulcerative colitis compared with those in familial adenomatous polyposis, and pouchitis was present only in patients who had had ulcerative colitis; the morphological features of pouchitis are similar to those seen in the colorectal mucosa in ulcerative colitis. Histochemical studies of mucin in the reservoirs of mucosa showed that there may have been a change from small intestinal mucin to colonic mucin.

In severe diffuse mucosal disease of the colon and rectum the entire large bowel may have to be removed. Several operations have been tried to preserve sphincter function and restore continuity of the bowel. Of these, restorative proctocolectomy with ileal reservoir is the most recent. ${ }^{1}$ The operation comprises total proctocolectomy, construction of a reservoir from the terminal ileum, and ileoanal anastomosis. The colon and about three quarters of the rectum are removed, and the mucosa stripped from the remaining rectal stump down to the dentate line. The reservoir of small intestine is then brought down through the denuded muscle tube and anastomosed to the anal canal at the dentate line. The length of rectal cuff left was about $8 \mathrm{~cm}$ in the early cases, but is now about $4 \mathrm{~cm}$ in some of the more recent cases. Various reservoir designs have been described. ${ }^{2} 3$ The procedure is covered by a temporary ileostomy that is usually closed about two months later, provided that all suture lines have healed.

Diffuse mucosal disease, in the form of ulcerative

Accepted for publication 29 January 1987 colitis or familial adenomatous polyposis, accounted for all but two of the 160 patients who have had proctocolectomy with ileal reservoir at St Mark's Hospital. Inflammation often occurs in the reservoir, and in a recent publication the incidence and severity of inflammation in 92 of these patients were described and the role of various physiological and pathological factors discussed. ${ }^{4}$ In this paper we present an account of the histopathological changes occurring in the mucosa of reservoirs, with particular reference to features seen on histochemical staining for mucins.

\section{Material and methods}

Mucosal biopsy specimens from the reservoirs of 92 patients who had undergone both stages of the operation were studied. In 78 cases the original indication was ulcerative colitis, and in 12 , familial adenomatous polyposis. Two operations were performed for function bowel disease, one a case of Hirschsprung's disease and the other a young adult with an ileostomy who had had necrotising enterocolitis as a child. Biopsy specimens were taken through a sigmoidoscope from each patient at intervals of six months to 


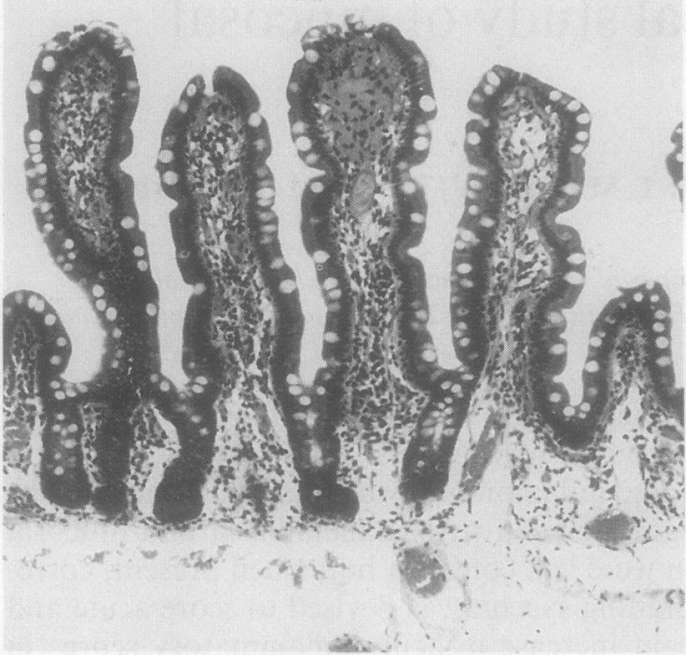

Fig 1 Biopsy of normal small intestinal mucosa from reservoir.

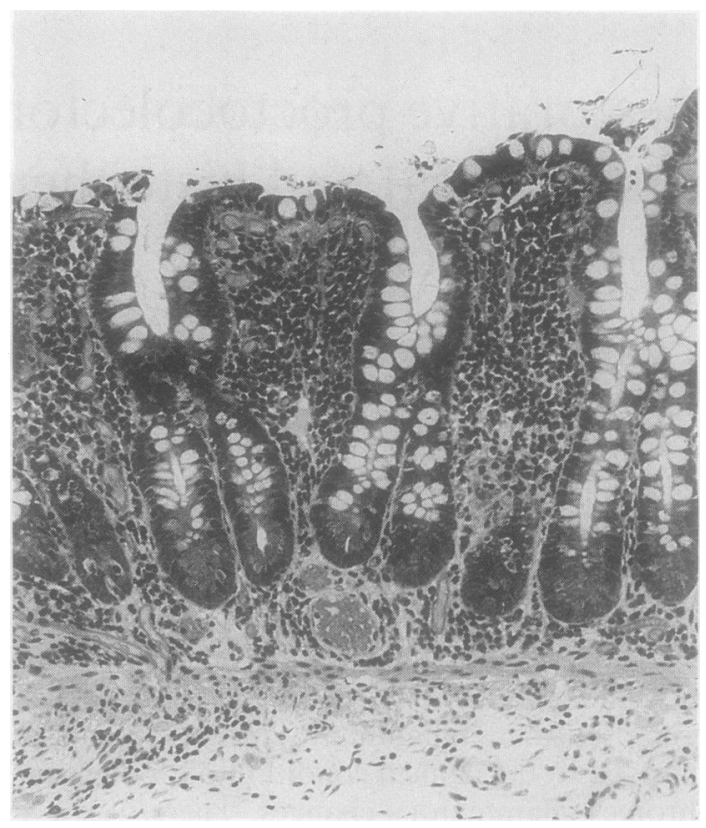

Fig 2 Mucosal biopsy specimen from reservoir showing partial villous atrophy and diffuse chronic inflammation of lamina propria. (Haematoxylin and eosin.)

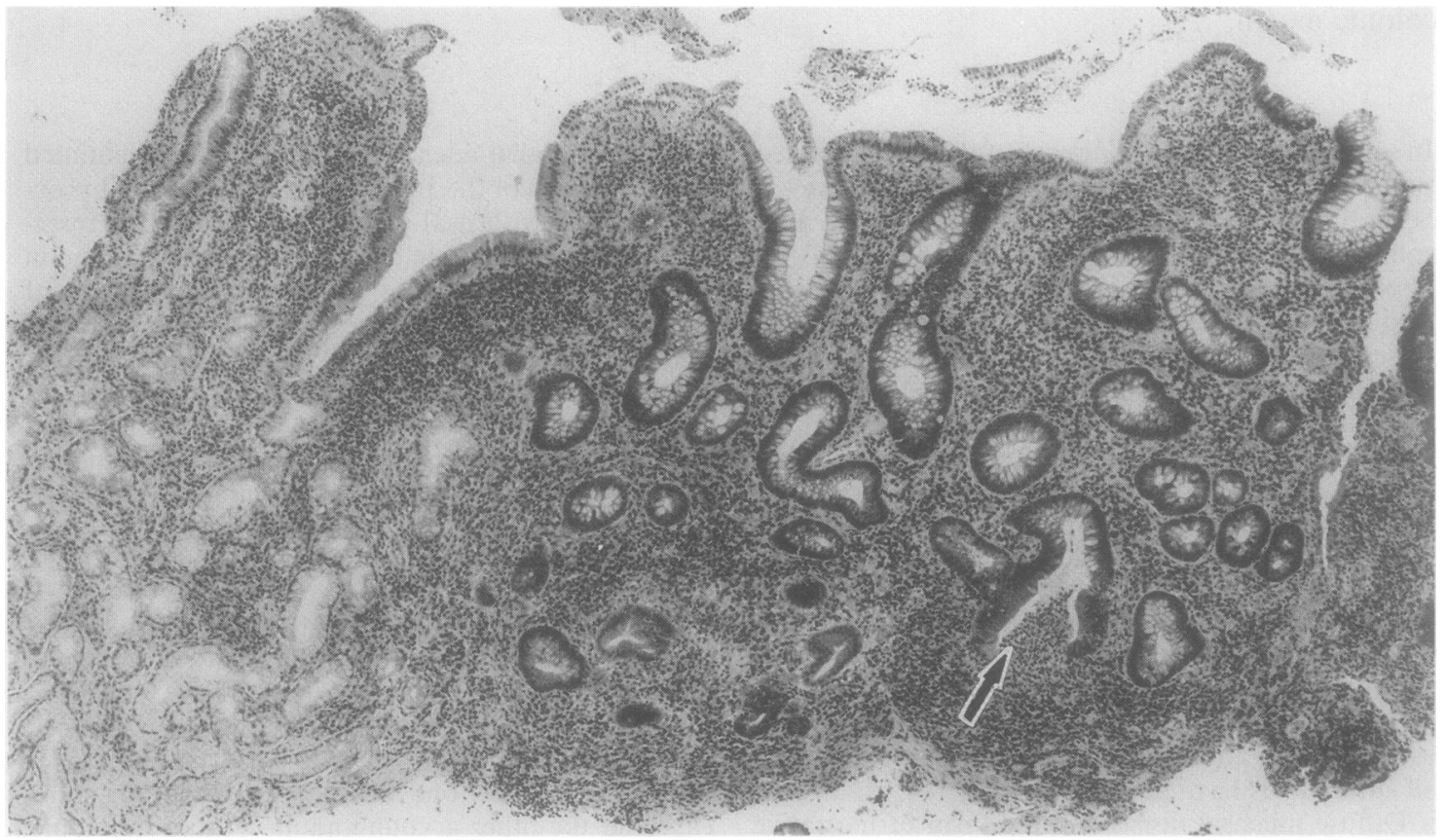

Fig 3 Mucosal biopsy specimen from reservoir showing pouchitis, with subtotal villous atrophy, pronounced crypt hyperplasia, diffuse chronic inflammation of lamina propria and pyloric metaplasia. There is active inflammation of superficial epithelium, and crypt abscess is shown (arrowed). (Haematoxylin and eosin.) 


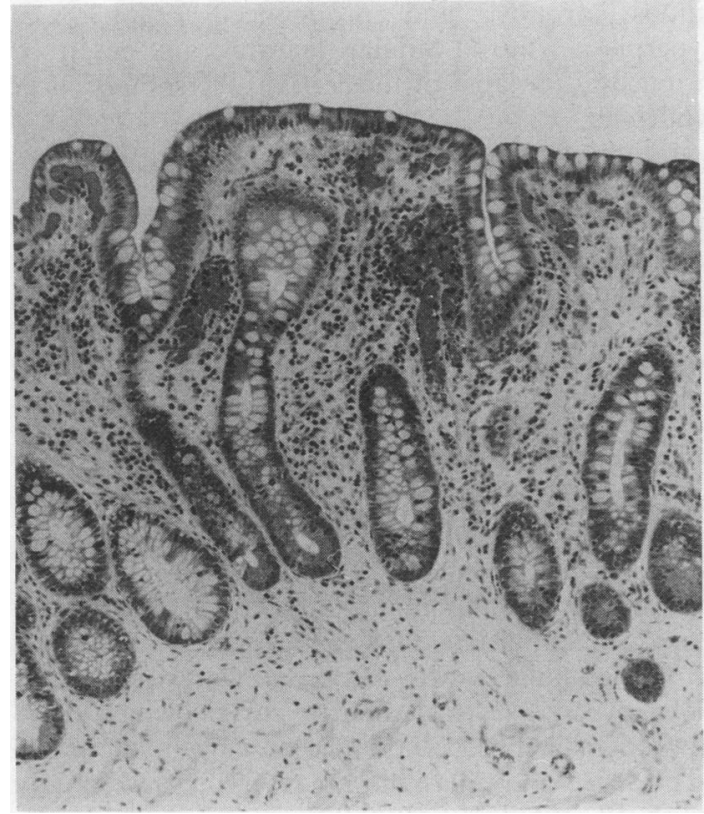

Fig 4 Mucosal biopsy specimen from reservoir with subtotal villous atrophy and pronounced crypt hyperplasia without noticeable inflammation. (Haematoxylin and eosin.)

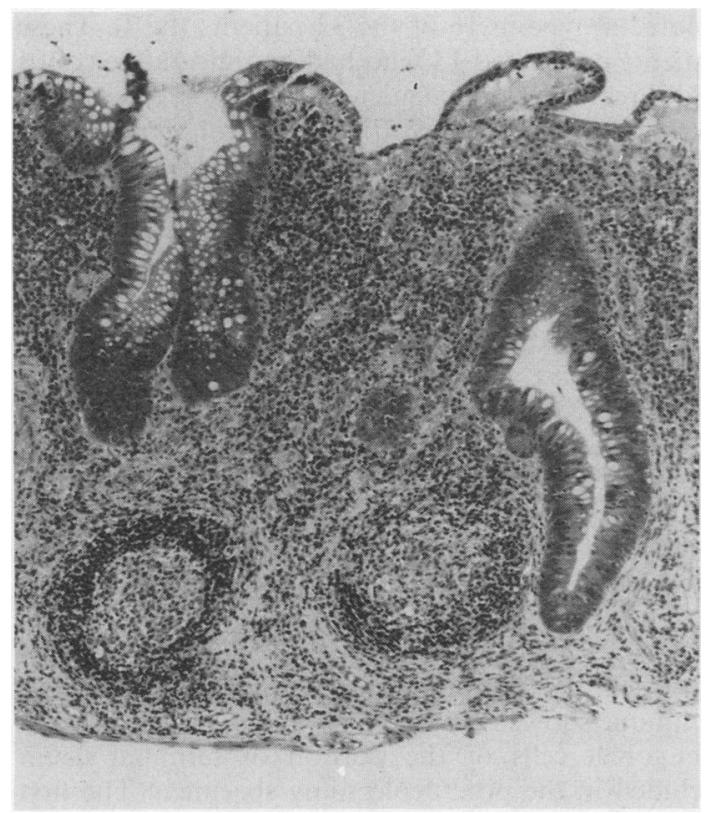

Fig 5 Mucosal biopsy specimen from reservoir with diffuse chronic inflammation, subtotal villous atrophy, crypt hyperplasia and crypt distortion. (Haematoxylin and eosin.) a year after closure of the ileostomy and were fixed in $10 \%$ buffered formol saline. The biopsy specimens were processed routinely in paraffin wax and stained with haematoxylin and eosin.

A subgroup of 32 patients, who had had at least three sequential mucosal biopsies of the reservoir, were further studied. In 25 , paraffin embedded blocks of ileum, which had been obtained at the time of the original proctocolectomy, were available. In addition to haematoxylin and eosin, high iron diamine-alcian blue (HID-AB) and periodate borohydride-potassium hydroxide-periodic acid Schiff (PB-KOH-PAS) stains were also performed. A modification of the method of Spicer, ${ }^{5}$ with ferric chloride at $60 \%$ concentration was used for HID-AB staining. All sections were cut and stained by the same person using the same technique. Each slide was graded by one observer (NAS) for blue predominance, a mixed pattern, or brown predominance. Assessment by PB-KOH-PAS was less satisfactory as normal small intestinal mucosa usually stains well, but a grading of none, mild, moderate or strong staining was used.

\section{Results}

Normal small intestinal mucosa was not usually found in mucosal biopsy specimens from the reservoirs; most showed chronic inflammatory changes. In only $12(13 \%)$ did the first mucosal biopsy specimen after ileostomy closure lack chronic inflammatory changes and villous architectural abnormality (fig 1 ). The chronic inflammatory cell infiltrate in the lamina propria included all cell types associated with chronic inflammation-lymphocytes, plasma cells, eosinophils and histiocytes-although often eosinophilic polymorphs were the first to accumulate. The chronic inflammatory changes were usually associated with some degree of villous atrophy (fig 2), which varied from mild and partial to almost total villous atrophy, common in cases with acute inflammation (fig 3). The degree of villous atrophy varied both in the sequential biopsy specimens and within the same specimen. When considerable acute inflammation was present, however, it was usually associated with extensive subtotal villous atrophy. Villous atrophy was always accompanied by pronounced crypt hyperplasia, producing an appearance similar to that of colonic mucosa (fig 4), and when this was associated with extensive chronic inflammation, the histological appearances were reminiscent of chronic ulcerative colitis in remission (fig 5).

In mucosal biopsy specimens from reservoirs which showed subtotal villous atrophy and crypt hyperplasia the histological appearances were like those of 
untreated coeliac disease (fig 2 ). There was, however, a notable difference in lymphocyte counts in the epithelium between biopsy specimens of small intestinal mucosa in coeliac disease and those of reservoir mucosa. Though intraepithelial lymphocytes are a prominent feature of untreated coeliac disease and are important in the assessment of response to a gluten free diet, the intraepithelial lymphocyte count in mucosal biopsy specimens from reservoirs was always less than $5 \%$ of epithelial cells. The lymphocyte count remained low even in the presence of active inflammation, although numerous polymorphs were usually present in the surface epithelium.

Other chronic changes seen in mucosal biopsy specimens from reservoirs were pyloric metaplasia and the histological features of mucosal prolapse. Pyloric metaplasia is a non-specific change that occurs in both small and large intestinal mucosa when it is affected by chronic inflammatory bowel disease; in these cases it was a rare feature of active inflammation in the pouch. It was seen in biopsy specimens in three patients in this study, all of whom had previously had acute inflammation (fig 3 ). In two other patients there was clinical evidence of mucosal prolapse within the reservoir. Histological examination of the mucosal biopsy specimens showed fibromuscular obliteration of the laminia propria,

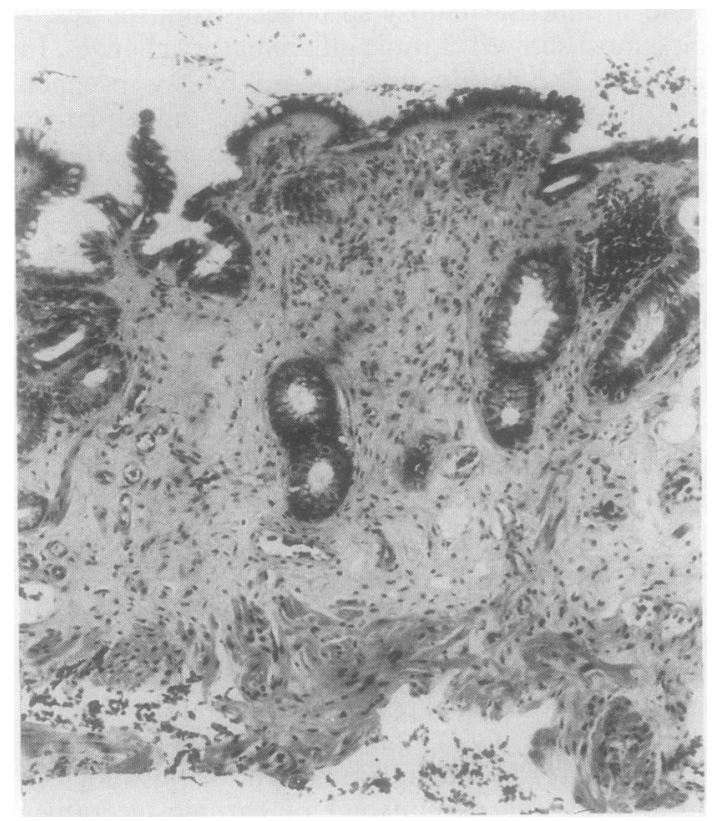

Fig 6 Mucosal biopsy specimen from reservoir in patient with clinical mucosal prolapse showing villous atrophy, fibromuscular obliteration of lamina propria, and crypt epithelial hyperplasia. (Haematoxylin and eosin.) villous atrophy, and pronounced crypt basal hyperplasia (fig 6). Similar features are described in mucosal prolapse of the rectum, the solitary ulcer syndrome. $^{6}$

Acute inflammatory changes and ulceration were seen less often. A patchy, superficial, acute inflammatory cell infiltrate in the epithelium was present in at least one biopsy specimen from a functioning reservoir in 50 cases of ulcerative colitis $(64 \%)$ and in six from cases of familial adenomatous polyposis $(50 \%)$. More extensive acute inflammatory cel infiltration, with crypt abscesses and ulceration, was seen in six cases $(7 \%)$, all of whom had had ulcerative colitis (fig 3). These histological changes, which were accompanied by pronounced chronic inflammation with villous atrophy, were always associated with pouchitis, manifest by systemic symptoms, the passage of at least six liquid motions per day, and macroscopic inflammation on sigmoidoscopy. ${ }^{4}$ Extensive active inflammation was associated with reactive epithelial hyperplasia (fig 3) in these cases in which ulcerative colitis had been diagnosed previously: there was no evidence of dysplasia in the reservoir. Two mildly dysplastic tubular adenomas were found in the mucosa in two cases of familial adenomatous polyposis.

Using the HID-AB technique we showed a considerable change from blue staining mucin of small intestinal goblet cell type to brown staining mucin of colorectal type in 16 of the 32 patients (fig 7). These patients comprised 13 who had had ulcerative colitis and three with familial adenomatous polyposis. They were all associated with histological evidence of chronic inflammation and villous atrophy. In three of the six with clinical pouchitis there was a change in the reservoir goblet cell mucin to a predominantly brown reacting product with HID-AB. With the PB$\mathrm{KOH}-\mathrm{PAS}$ staining sequence, there was a definite increase in PAS staining between ileal control and reservoir mucosa in only six patients, all of whom had had ulcerative colitis.

The mucosa of the terminal ileum at the time of the original proctocolectomy was histologically normal in 24 of the 25 patients for whom tissue was available. There was no evidence of villous atrophy, crypt hyperplasia, or inflammation. In only one case, a 35 year old man with ulcerative colitis, was there clinically important inflammation in the terminal ileal mucosa at the time of proctocolectomy. The HID-AB technique showed a predominantly brown reaction in the goblet cells of the portion of terminal ileum included in the proctocolectomy specimen. The first mucosal biopsy specimen of the reservoir showed a predominantly blue reaction, but later specimens showed a reversion to a brown staining mucin of goblet cell type, indicating an increased sulphate content. 

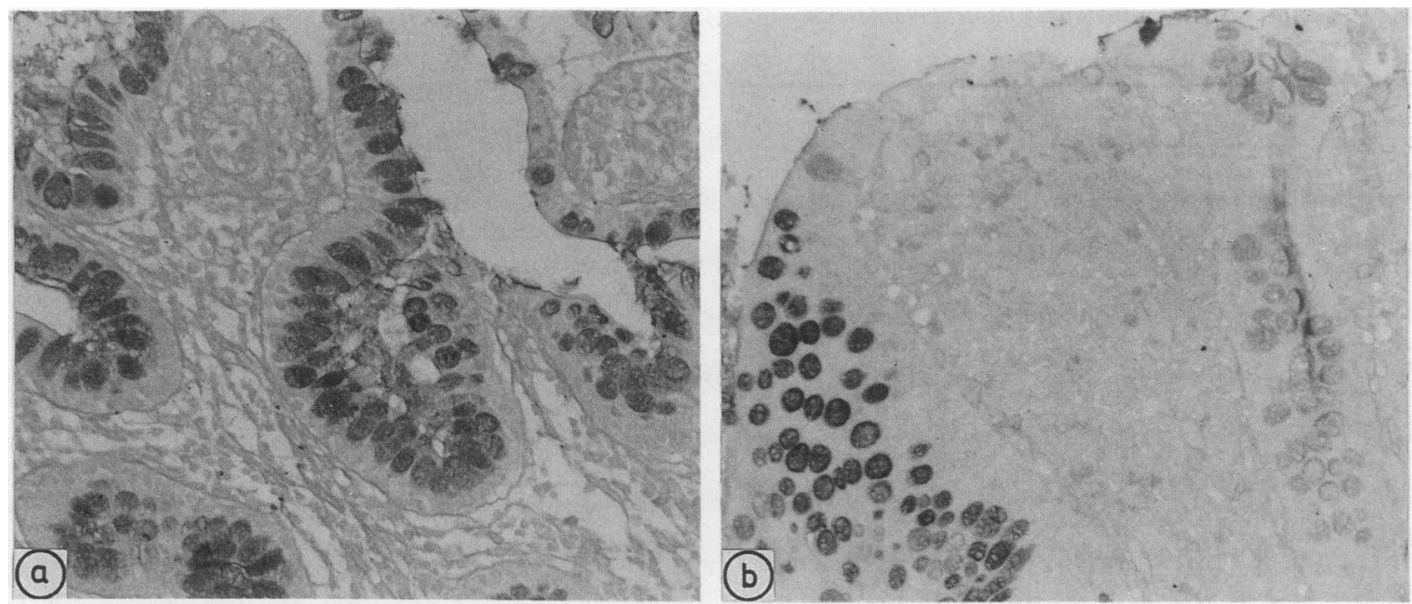

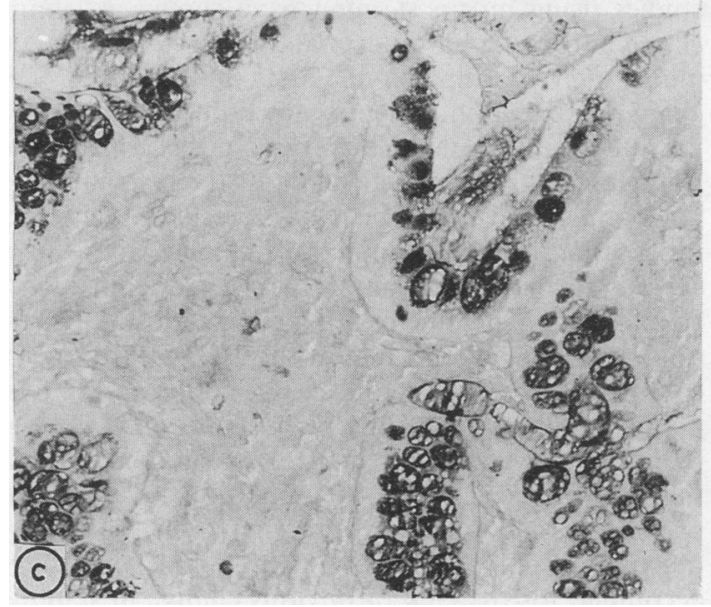

\section{Discussion}

Little attention has been paid to the histopathological changes that occur in the small intestinal mucosa in reservoirs after restorative proctocolectomy. As the operation becomes more common pathologists will be increasingly confronted by mucosal biopsy specimens from the reservoirs, and the findings of this prospective study may help them in their assessments. We have shown that histological changes, which are almost always present, are predominantly chronic, and include both inflammation and villous atrophy. In cases with extensive acute and chronic inflammatory changes there is such pronounced villous atrophy and crypt hyperplasia and distortion, that the mucosa resembles that of the large intestine in chronic ulcerative colitis. In our study only one of 25 cases showed histological abnormality in the ter- minal ileal mucosa at the time of proctocolectomy, and therefore the changes seen in the reservoir mucosa seem to be the direct result of creation of the reservoir.

In a previous study, in which clinical and sigmoidoscopic features were correlated with histology of the reservoir mucosa, we introduced a histological grading system for the assessment of severity of inflammation and this is reproduced in the table. ${ }^{4}$ Scores for acute inflammation correlated with the degree of macroscopic inflammation seen on sigmoidoscopy, and with the frequency of defaecation, and were lower in those patients with familial adenomatous polyposis compared with those who had ulcerative colitis. There was, however, no significant difference in the score for chronic inflammation in patients with ulcerative colitis, compared with those with familial adenomatous polyposis. ${ }^{4} \mathrm{~A}$ histological 
Table Scoring system for histopathological changes in reservoir muscosa ${ }^{4}$

\begin{tabular}{ll}
\hline Histological feature & Score \\
\hline $\begin{array}{l}\text { Acute } \\
\text { Polymorph infiltration: }\end{array}$ & \\
$\quad$ None & 0 \\
$\quad$ Mild and patchy infiltrate in the surface epithelium & 1 \\
$\quad$ Moderate with crypt abscesses & 2 \\
$\quad$ Severe with crypt abscesses & 3 \\
Ulceration: & \\
$\quad$ None & 0 \\
$\quad$ Mild superficial & 1 \\
Moderate & 2 \\
$\quad$ Extensive & 3 \\
Chronic & \\
Chronic inflammatory cell infiltration: & \\
$\quad$ None & \\
Mild and patchy & 0 \\
Moderate & 1 \\
Severe & 2 \\
Villous atrophy: & 3 \\
$\quad$ None & \\
Minor abnormality of villous architecture & \\
Partial villous atrophy & \\
Subtotal villous atrophy & 1 \\
\hline
\end{tabular}

grading for acute changes of four or more indicated active pouchitis, and correlated with the clinical criteria of this condition. ${ }^{4}$

We have seen pouchitis only in those reservoirs created for ulcerative colitis, but as these comprise over $80 \%$ of the total it is difficult to draw definite conclusions about the apparent absence of pouchitis from those reservoirs created for familial adenomatous polyposis. More recent figures in our series show 10 patients with pouchitis, all of whom had had ulcerative colitis, out of a total of 152 cases (126 ulcerative colitis, 24 familial adenomatous polyposis, two miscellaneous). Furthermore, we have shown that there is considerably more acute inflammatory change in those reservoirs constructed from ulcerative colitis. ${ }^{4}$ In the Kock continent ileostomy, where an ileal reservoir is constructed with an abdominal stoma, definite acute inflammation is also seen only in cases of ulcerative colitis. ${ }^{7}$ Current evidence suggests, therefore, that severe acute inflammation, including pouchitis, is a disease confined to reservoirs constructed for ulcerative colitis and that it does not occur in ileal reservoirs constructed for familial adenomatous polyposis.

Although we have seen hyperplastic epithelial changes in cases with active inflammation, we have not seen dysplasia in the mucosa of those reservoirs constructed for ulcerative colitis. None of the reservoirs, however, has been in situ for more than 10 years. Two mildly dysplastic tubular adenomas were found in two reservoirs constructed for familial adenomatous polyposis. The apparent colonic metaplasia in the reservoir mucosa may lead to an increased propensity for adenomas to develop in the reservoir, and there is a case report of multiple adenomas (about 30 ) occurring in a Kock continent ileostomy. ${ }^{8}$ Adenomas may, however, also be seen in the otherwise normal small intestinal mucosa in familial adenomatous polyposis. ${ }^{9}$

Histochemical studies using mucin staining were performed on a subgroup of 32 patients, 26 with ulcerative colitis and six with familial adenomatous polyposis. The goblet cells of the terminal ileum stain mainly blue with HID-AB. ${ }^{10}$ This indicates the presence of sialic acid with little or no sulphate. We have found that there is some sulphomucin present in the normal small intestine, especially in the epithelium overlying the lymphoid aggregates of Peyer's patches, and this emphasises the importance of using control tissues from the same patient. Our histochemical studies using mucin staining have shown a definite change from small intestinal mucin to colorectal mucin rich in sulphate in $50 \%$ of the cases. The change was seen in patients with both ulcerative colitis and familial adenomatous polyposis. This mucin change seems to be independent of the original diagnosis and probably occurs as a result of chronic, rather than acute, inflammatory change. It is, however, known to be unstable. A change from highly sulphated colonic mucin to less sulphated small intestinal mucin (brown to blue with HID-AB) occurs in colonic mucosa in acute ulcerative colitis and in dysplasia in ulcerative colitis. ${ }^{11}$ In some of our cases there may have been a change to colonic mucin, but that the onset of active inflammation reversed this process in a similar way to that seen in colonic mucosa in active ulcerative colitis. This may have accounted for the lack of obvious change in staining in three of the cases with clinical and histological evidence of pouchitis.

Previous studies have shown that the onset of acute inflammatory changes in the reservoir mucosa does not correlate with the type of reservoir, its emptying characteristics, or its volume. ${ }^{412}$ This suggests that stasis alone is not a factor in the development of pouchitis. Recent bacteriological studies have not found a consistent correlation between aerobic or anaerobic bacterial counts and inflammation, ${ }^{13}$ although one study showed some correlation between concentrations of aerobic organisms and inflammation, ${ }^{14}$ and another study showed a significant inverse correlation between volatile fatty acids (which reflect anaerobic bacterial activity) and villous atrophy in the reservoir mucosa. ${ }^{15}$ This finding suggests that high counts of anaerobic bacteria may protect the reservoir mucosa. Some patients with pouchitis do, however, benefit from metronidazole ${ }^{13}$ which contradicts this observation.

The creation of a neorectum, which becomes fur- 
ther modified by a process of colonic metaplasia, may create an environment that favours the development of a condition similar to ulcerative colitis in the ileal reservoir. This hypothesis is supported by morphological changes in the pouch, which bear a striking resemblance to ulcerative colitis, and the fact that an inflammatory process does occur in small intestinal mucosa in ulcerative colitis. Currently there is no evidence to suggest that bacteriological or physical factors are implicated in the aetiology of pouchitis.

We thank Ms Jill Maybee for photographic help.

\section{References}

I Parks AG, Nicholls RJ. Proctocolectomy without ileostomy for ulcerative colitis. $\mathrm{Br}$ Med J 1978;2:85-8.

2 Utsunomiya J, Iwama T, Imago $\mathbf{M}$, et al. Total colectomy, mucosal proctectomy and ileoanal anastomosis. Dis Colon Rectum 1980;23:459-66.

3 Nicholls RJ, Pezim ME. Restorative proctocolectomy with ileal reservoir for ulcerative colitis and familial adenomatous polyposis: a comparison of three reservoir designs. Br J Surg 1985;72:470-4.

4 Moskowitz RL, Shepherd NA, Nicholls RJ. An assessment of inflammation in the reservoir after restorative proctocolectomy with ileoanal ileal reservoir. International Journal of Colorectal Disease 1986;1:167-74.
5 Spicer SS. The use of various cationic reagents in histochemical differentiation of mucopolysaccharides. Am J Clin Pathol 1961;5:393-407.

6 Madigan MR, Morson BC. Solitary ulcer of the rectum. Gut 1969;10:871-81.

7 Hulten L, Svaninger G. Facts about the Kock continent ileostomy. Dis Colon Rectum 1984;27:553-7.

8 Beart RW Jr, Fleming CR, Banks PM. Tubulovillous adenomas in a continent ileostomy after proctocolectomy for familial polyposis. Dig Dis Sci 1982;27:553-6.

9 Hamilton SR, Bussey HJR, Mendelsohn G, et al. Ileal adenomas after colectomy in nine patients with adenomatous polyposis coli/Gardner's syndrome. Gastroenterology 1979;77:1252-7.

10 Filipe MI. Mucins in the human gastrointestinal tract: a review. Invest Cell Pathol 1979;2:195-216.

11 Jass JR, England J, Miller K. Value of mucin histochemistry in follow up surveillance of patients with long standing ulcerative colitis. J Clin Pathol 1986;39:393-8.

12 Dozois RR. Pathophysiology. Symposium; restorative proctocolectomy with ileal reservoir. International Journal of Colorectal Disease 1986;1:14-5.

13 Moskowitz RL. Pathophysiology. Symposium; restorative proctocolectomy with ileal reservoir. International Journal of Colorectal Disease 1986;1:16-7.

14 Nicholls RJ, Belliveau P, Neill M, Wilks M, Tabaqchali S. Restorative proctocolectomy with ileal reservoir: a pathophysiological assessment. Gut 1981;22:462-8.

15 Nasmyth DG, Godwin PGR, Dixon MF, Williams NS, Johnston $D$. The relationship between mucosal structure and intestinal flora in ileal reservoirs. Br J Surg 1985;72:S129.

Requests for reprints to: Dr NA Shepherd, Department of Pathology, St Mark's Hospital, City Road, London ECIV 2PS, England. 